\title{
Local Model-Checking of Modal Mu-Calculus on Acyclic Labeled Transition Systems
}

\author{
Radu Mateescu \\ INRIA Rhône-Alpes / VASY, 655, avenue de l'Europe \\ F-38330 Montbonnot Saint Martin, France \\ Radu.Mateescu@inria.fr
}

\begin{abstract}
Model-checking is a popular technique for verifying finitestate concurrent systems, whose behaviour can be modeled using Labeled Transition Systems (LTSs). In this paper, we study the model-checking problem for the modal $\mu$-calculus on acyclic LTSs. This has various applications of practical interest such as trace analysis, log information auditing, run-time monitoring, etc. We show that on acyclic LTSs, the full $\mu$-calculus has the same expressive power as its alternation-free fragment. We also present two new local model-checking algorithms based upon a translation to boolean equation systems. The first algorithm handles $\mu$-calculus formulas $\varphi$ with alternation depth $\operatorname{ad}(\varphi) \geq 2$ and has time complexity $O\left(|\varphi|^{2} \cdot(|S|+|T|)\right)$ and space complexity $O\left(|\varphi|^{2} \cdot|S|\right)$, where $|S|$ and $|T|$ are the number of states and transitions of the acyclic LTS and $|\varphi|$ is the number of operators in $\varphi$. The second algorithm handles formulas $\varphi$ with alternation depth $\operatorname{ad}(\varphi)=1$ and has time complexity $O(|\varphi| \cdot(|S|+|T|))$ and space complexity $O(|\varphi| \cdot|S|)$.
\end{abstract}

\section{Introduction}

Model-checking 3 is a popular approach for efficiently verifying the correctness of concurrent finite-state systems. This approach proceeds by translating the system into a finite model, represented as a state transition graph or a Labeled Transition System (LTS), on which the desired correctness properties, expressed in temporal logic, are verified using specific model-checking algorithms. According to the way in which they handle the construction of the LTS, model-checking algorithms can be divided in two classes: global algorithms, which require to construct the LTS completely before starting the verification, and local algorithms, which allow to construct the LTS in a demand-driven way during verification. The latter algorithms are able to detect errors even if the LTs cannot be entirely constructed (e.g., because of insufficient computing resources).

Although model-checking has been mainly used for verifying concurrent systems (communication protocols, distributed applications, hardware architectures), the underlying techniques and algorithms are useful in other contexts as well. In particular, several problems related to the analysis of sequential systems can be formulated as model-checking problems on single trace LTSs: intrusion detection by auditing of log file information, as in the UsTAT rule-based expert

J.-P. Katoen and P. Stevens (Eds.): TACAS 2002, LNCS 2280, pp. 281295 2002

(C) Springer-Verlag Berlin Heidelberg 2002 
system 13, in which security properties of log files are encoded as state transition diagrams; trace analysis for program debugging, as in the OPIUM trace analyzer for Prolog [8], which uses a dedicated language for describing trace queries; and run-time monitoring by observation of event traces in real-time, as in the Motel monitoring system [6], which uses LTL 19] for expressing test requirements and for synthesizing observers. It appears that, when analyzing sequential systems, existing modal-checking algorithms can be optimized significantly, especially by reducing their memory consumption, which is crucial for scaling up to larger systems. Therefore, optimizing the performance of modelchecking algorithms on sequential systems becomes an interesting issue, with applications in all aforementioned domains.

In this paper, we consider the problem of model-checking temporal properties on acyclic LTSs (AlTSs), of which traces are a particular case. As regards the property specification language, we adopt the modal $\mu$-calculus [15], a powerful fixed point-based temporal logic that subsumes virtually all temporal logics defined in the literature (in-depth presentations of modal $\mu$-calculus can be found in $\left[\begin{array}{l|l|l|l|l|l|}18 & 22\end{array}\right)$. Various global algorithms [94|5] and local algorithms [16. 1241721 have been proposed for model-checking $\mu$-calculus formulas on arbitrary LTSs. However, as far as we know, no attempt has been made to optimize these algorithms in the case of AlTSs.

Our results concern both the expressiveness of $\mu$-calculus interpreted on ALtSs, and the underlying model-checking algorithms. We first show that the full modal $\mu$-calculus interpreted on Altss has the same expressive power as its alternation-free fragment [9]. Our result is based upon a succinct translation (quadratic blow-up of the formula size) from full $\mu$-calculus to guarded $\mu$-calculus [15], followed by a reduction to alternation-free $\mu$-calculus. Together with the linear-time complexity results for alternation-free $\mu$-calculus [5], this yields a model-checking procedure for the full $\mu$-calculus on ALTSs which is quadratic in the size of the formula (number of operators) and linear in the size of the Alts (number of states and transitions).

We also propose two local model-checking algorithms for $\mu$-calculus on ALTss based upon a translation to boolean equation systems (BESs) 18. The first algorithm handles full $\mu$-calculus formulas and has a time complexity $O\left(|\varphi|^{2} \cdot(|S|+|T|)\right)$ and a space complexity $O\left(|\varphi|^{2} \cdot|S|\right)$, where $|\varphi|$ is the size of the formula and $|S|,|T|$ are the number of states and transitions in the Alts. The second algorithm handles only alternation-free formulas and has a time complexity $O(|\varphi| \cdot(|S|+|T|))$ and a space complexity $O(|\varphi| \cdot|S|)$. Both algorithms exploit the particular structure of the underlying BES to avoid storing AlTs transitions and thus to achieve a lower space complexity than existing local model-checking algorithms [1,24,17,7] executed on AlTss.

The paper is organized as follows. Section 2 defines the modal $\mu$-calculus and its guarded fragment, and presents the simplification results for $\mu$-calculus formulas on ALTSs. Section [3 describes the local model-checking algorithms for full $\mu$-calculus and alternation-free $\mu$-calculus on ALTSs. Section 4 gives some concluding remarks and directions for future work. 


\section{Modal Mu-Calculus and Acyclic LTSs}

In this section we study the expressiveness of $\mu$-calculus formulas interpreted on acyclic LTSs, our goal being to simplify formulas as much as possible in order to increase the efficiency of model-checking algorithms. We first define the syntax and semantics of the modal $\mu$-calculus, then we propose a succinct translation of the $\mu$-calculus to its guarded fragment, and finally we present the simplification results obtained.

\subsection{Syntax and Semantics}

As interpretation models, we consider labeled transition systems (LTSs), which are suitable for action-based description languages such as process algebras. An LTS is a tuple $M=\left(S, A, T, s_{0}\right)$, where: $S$ is a (finite) set of states; $A$ is a (finite) set of actions; $T \subseteq S \times A \times S$ is the transition relation; and $s_{0} \in S$ is the initial state. A transition $\left(s_{1}, a, s_{2}\right) \in T$ (also noted $s_{1} \stackrel{a}{\rightarrow} s_{2}$ ) means that the system can move from state $s_{1}$ to state $s_{2}$ by performing action $a$. The notation $s_{1} \stackrel{*}{\rightarrow} s_{2}$ means that there exists a sequence of (0 or more) transitions leading from $s_{1}$ to $s_{2}$. All states in $S$ are reachable from $s_{0}$ via sequences of transitions in $T\left(s_{0} \stackrel{*}{\rightarrow} s\right.$ for all $s \in S$ ). If $T$ does not contain cycles, $M$ is called an acyclic LTs (Alts). In the sequel, we assume the existence of an $\operatorname{LTS} M=\left(S, A, T, s_{0}\right)$ on which all temporal logic formulas will be interpreted.

The $\mu$-calculus variant we consider here (see Table 1 ) is slightly extended w.r.t. the original one [15]: instead of simple actions $a \in A$, the modalities contain action formulas $\alpha$ built from actions and standard boolean operators (derived connectives are $\mathrm{F}=a \wedge \neg a$ for some $a \in A, \mathrm{~T}=\neg \mathrm{F}, \alpha_{1} \vee \alpha_{2}=\neg\left(\neg \alpha_{1} \wedge \neg \alpha_{2}\right)$, etc.). State formulas $\varphi$ are built from propositional variables $X \in \mathcal{X}$, standard boolean operators, possibility and necessity modal operators $\langle\alpha\rangle \varphi$ and $[\alpha] \varphi$, minimal and maximal fixed point operators $\mu X . \varphi$ and $\nu X . \varphi$. The $\mu$ and $\nu$ operators act as binders for variables in a way similar to quantifiers in first-order logic. A formula $\varphi$ without free occurrences of variables is closed. In the sequel, we assume that state formulas are in normal form, i.e., all propositional variables bound by fixed point operators have distinct names. We use the symbol $\sigma$ to denote $\mu$ or $\nu$.

The interpretation $\llbracket \alpha \rrbracket \subseteq A$ of an action formula $\alpha$ yields the set of LTS actions satisfying $\alpha$. The interpretation $\llbracket \varphi \rrbracket \rho \subseteq S$ of a state formula $\varphi$, where $\rho: \mathcal{X} \rightarrow 2^{S}$ is a propositional context assigning state sets to variables, yields the set of LTS states satisfying $\varphi$ in the context $\rho(\rho[U / X]$ denotes a context identical to $\rho$ except for variable $X$, which is assigned state set $U)$. The $\langle\alpha\rangle \varphi$ and $[\alpha] \varphi$ modalities characterize the states for which some, respectively all outgoing transitions whose actions satisfy $\alpha$ lead to states satisfying $\varphi$. The $\mu X . \varphi$ and $\nu X . \varphi$ formulas characterize the states satisfying the minimal, respectively maximal solution (over $2^{S}$ ) of the equation $X=\varphi$. For closed state formulas $\varphi$, we simply write $\llbracket \varphi \rrbracket$, since the interpretation of these formulas does not depend upon any propositional context. An $\operatorname{LTS} M=\left(S, A, T, s_{0}\right)$ satisfies a closed state formula $\varphi$ (notation $M \models \varphi$ ) iff $\llbracket \varphi \rrbracket=S$. 
Table 1. Syntax and semantics of the modal $\mu$-calculus

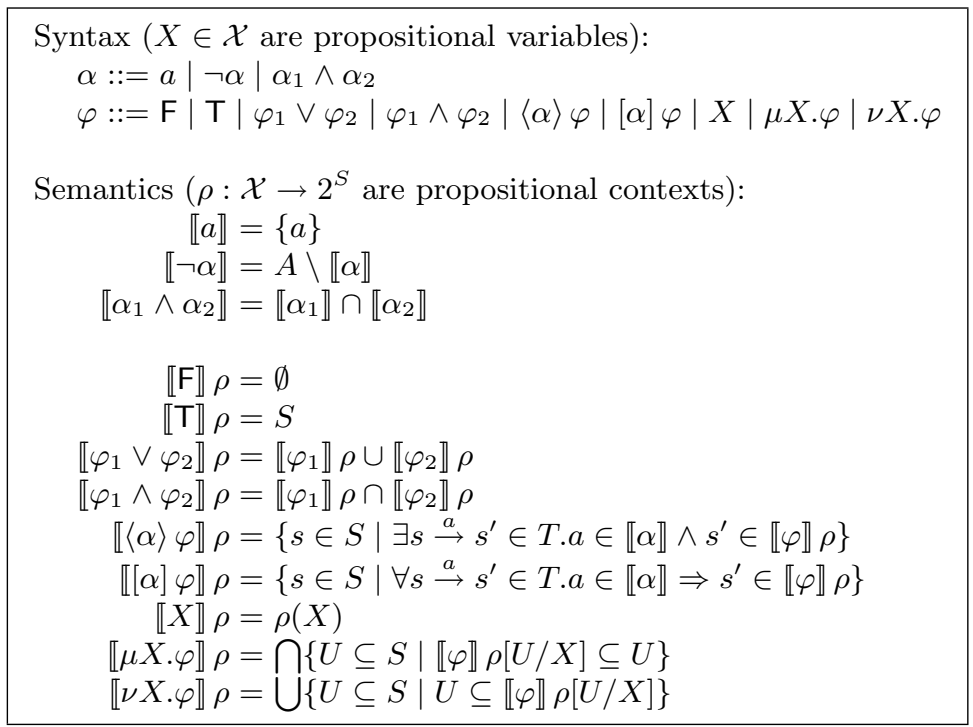

\subsection{Reduction of Full Mu-Calculus to Guarded Mu-Calculus}

In order to simplify the interpretation of modal $\mu$-calculus on ALtss, we must first translate all fixed point formulas $\sigma X . \varphi$ in guarded form [15|23]: all free occurrences of $X$ in $\varphi$ must be guarded, i.e., in the scope of a \langle\rangle or [ ] modality. The translations proposed in [15]23] require repeated transformations of subformulas in conjunctive normal form, leading to an exponential blow-up of the formula. We present below a more succinct translation in guarded form, which (by using factorization of common subformulas) yields only a quadratic blow-up. This translation is purely syntactic, i.e., it does not depend upon the structure of the LTS $M=\left(S, A, T, s_{0}\right)$ on which formulas are interpreted.

Definition 1 (guarded and weakly guarded formulas). Let $\varphi$ be a state formula and $\mathcal{X}$ be a set of variables. $\varphi$ is called guarded, resp. weakly guarded w.r.t. $\mathcal{X}$ iff it satisfies the predicate $g(\varphi, \mathcal{X})$, resp. $w g(\varphi, \mathcal{X})$, defined inductively below. $\varphi$ is called guarded iff it satisfies $g(\varphi, \emptyset)$.

\begin{tabular}{|l||l|l|}
\hline \multicolumn{1}{|c|}{$\varphi$} & \multicolumn{1}{c|}{$g(\varphi, \mathcal{X})$} & \multicolumn{1}{c|}{$w g(\varphi, \mathcal{X})$} \\
\hline \hline $\mathrm{F}, \mathrm{T}$ & $\mathrm{T}$ & $\mathrm{T}$ \\
\hline$\varphi_{1} \vee \varphi_{2}, \varphi_{1} \wedge \varphi_{2}$ & $g\left(\varphi_{1}, \mathcal{X}\right) \wedge g\left(\varphi_{2}, \mathcal{X}\right)$ & $w g\left(\varphi_{1}, \mathcal{X}\right) \wedge w g\left(\varphi_{2}, \mathcal{X}\right)$ \\
\hline$\langle\alpha\rangle \varphi,[\alpha] \varphi$ & $g(\varphi, \emptyset)$ & $g(\varphi, \emptyset)$ \\
\hline$X$ & $X \notin \mathcal{X}$ & $\mathrm{T}$ \\
\hline$\mu X . \varphi, \nu X . \varphi$ & $g(\varphi, \mathcal{X} \cup\{X\})$ & $g(\varphi, \mathcal{X} \cup\{X\})$ \\
\hline
\end{tabular}


Intuitively, a formula $\varphi$ weakly guarded w.r.t. $\{X\}$ allows unguarded occurrences of $X$ only at top-level, i.e., outside any fixed point subformula of $\varphi$. The formula below, obtained by translating in $\mu$-calculus the PDL [11] regular modality $\left\langle\left(a \mid b^{*}\right)^{*} . c\right\rangle \mathrm{T}$, is unguarded w.r.t. $\{X\}$ :

$$
\varphi_{1}=\mu X .(\langle c\rangle \mathrm{\top} \vee\langle a\rangle X \vee \mu Y .(X \vee\langle b\rangle Y))
$$

To make this formula guarded, we must eliminate the unguarded occurrence of $X$ contained in the $\mu Y$-subformula. The first step is to bring this occurrence at the top-level of the body of $\varphi_{1}$. This is done by unfolding the $\mu Y$-subformula, resulting in the formula below, whose body is weakly guarded w.r.t. $\{X\}$ :

$$
\varphi_{2}=\mu X .(\langle c\rangle \mathrm{T} \vee\langle a\rangle X \vee(X \vee\langle b\rangle \mu Y .(X \vee\langle b\rangle Y)))
$$

The second step is to eliminate the top-level unguarded occurrence of $X$. This is done using the transformation below, which replaces, in a formula $\mu X . \varphi$ (resp. $\nu X . \varphi)$, all unguarded occurrences of $X$ at the top-level of $\varphi$ by $\mathrm{F}$ (resp. T).

Definition 2 (flattening). Let $\varphi$ be a state formula, $X$ be a variable, and $\sigma \in$ $\{\mu, \nu\}$. The formula $f(\varphi, X, \sigma)$ defined inductively below is called the flattening of $\varphi$ w.r.t. $X$.

\begin{tabular}{|l|l||l|l|}
\hline \multicolumn{1}{|c|}{$\varphi$} & \multicolumn{1}{|c|}{$f(\varphi, X, \sigma)$} & \multicolumn{1}{c|}{$\varphi$} & \multicolumn{1}{c|}{$f(\varphi, X, \sigma)$} \\
\hline \hline $\mathrm{F}$ & $\mathrm{F}$ & $\mathrm{T}$ & $\mathrm{T}$ \\
\hline$\varphi_{1} \vee \varphi_{2}$ & $f\left(\varphi_{1}, X, \sigma\right) \vee f\left(\varphi_{2}, X, \sigma\right)$ & $\varphi_{1} \wedge \varphi_{2}$ & $f\left(\varphi_{1}, X, \sigma\right) \wedge f\left(\varphi_{2}, X, \sigma\right)$ \\
\hline$\langle\alpha\rangle \varphi$ & $\langle\alpha\rangle \varphi$ & {$[\alpha] \varphi$} & {$[\alpha] \varphi$} \\
\hline$Y$ & $Y($ if $Y \neq X)$ & $X$ & if $\sigma=\mu$ then $\mathrm{F}$ else $\mathrm{T}$ \\
\hline$\mu Y . \varphi$ & $\mu Y . \varphi$ & $\nu Y . \varphi$ & $\nu Y . \varphi$ \\
\hline
\end{tabular}

By flattening the formula $\varphi_{2}$, we obtain the guarded formula below:

$$
\varphi_{3}=\mu X .(\langle c\rangle \mathrm{T} \vee\langle a\rangle X \vee(\mathrm{F} \vee\langle b\rangle \mu Y .(X \vee\langle b\rangle Y)))
$$

This formula can be translated back into the PDL modality $\left\langle\left(a \mid\left(b . b^{*}\right)\right)^{*} . c\right\rangle \mathrm{T}$, which is equivalent to the initial formula $\left\langle\left(a \mid b^{*}\right)^{*} . c\right\rangle \mathrm{T}$.

The following results state that flattening of fixed point formulas does not change their interpretation.

Lemma 1. Let $\varphi$ be a state formula, $X$ be a variable, and $U \subseteq S$. Then:

$$
\llbracket f(\varphi, X, \nu) \rrbracket \rho[U / X] \cap U \subseteq \llbracket \varphi \rrbracket \rho[U / X] \subseteq \llbracket f(\varphi, X, \mu) \rrbracket \rho[U / X] \cup U
$$

for any propositional context $\rho$.

Proposition 1. Let $\sigma X . \varphi$ be a state formula. Then:

$$
\llbracket \sigma X . \varphi \rrbracket \rho=\llbracket \sigma X . f(\varphi, X, \sigma) \rrbracket \rho
$$

for any propositional context $\rho$. 
Proof. We show only the case $\sigma=\mu$, the other case being similar. Since some occurrences of $X$ in $\varphi$ have been replaced by $\mathrm{F}$ in $f(\varphi, X, \mu)$, it follows by monotonicity that $\llbracket \mu X . f(\varphi, X, \mu) \rrbracket \rho \subseteq \llbracket \mu X . \varphi \rrbracket \rho$. To show the converse, let $U \subseteq S$ such that $\llbracket f(\varphi, X, \mu) \rrbracket \rho[U / X] \subseteq U$. Using Lemma 1 this implies $\llbracket \varphi \rrbracket \rho[U / X] \subseteq \llbracket f(\varphi, X, \mu) \rrbracket \rho[U / X] \cup U=U$. This further implies $\{U \subseteq S \mid \llbracket f(\varphi, X, \mu) \rrbracket \rho[U / X] \subseteq U\} \subseteq\{U \subseteq S \mid \llbracket \varphi \rrbracket \rho[U / X] \subseteq U\}$, which by interpretation of formulas (Table 1) yields $\llbracket \mu X . \varphi \rrbracket \rho=\bigcap\{U \subseteq S \mid \llbracket \varphi \rrbracket \rho[U / X] \subseteq$ $U\} \subseteq \bigcap\{U \subseteq S \mid \llbracket f(\varphi, X, \mu) \rrbracket \rho[U / X] \subseteq U\}=\llbracket \mu X . f(\varphi, X, \mu) \rrbracket \rho$.

The transformation defined below, consisting of two mutually recursive functions $t$ and $t^{\prime}$, reduces state formulas to guarded form. These functions implement the transformation outlined in the previous example when $\varphi_{1}$ was translated to $\varphi_{3}$ : for every fixed point formula $\sigma X . \varphi$, the unguarded occurrences of $X$ are brought to the top-level of $\varphi$ using $t^{\prime}(\varphi)$ and then they are eliminated by flattening using $f\left(t^{\prime}(\varphi), X, \sigma\right)$. By applying $t^{\prime}(\varphi)$, the fixed point subformulas of $\varphi$ that are not in the scope of a modality are unfolded $\left(\varphi^{\prime}\left[\varphi^{\prime \prime} / X\right]\right.$ denotes the syntactic substitution of the free occurrences of $X$ in $\varphi^{\prime}$ by $\left.\varphi^{\prime \prime}\right)$.

Definition 3 (translation to guarded $\boldsymbol{\mu}$-calculus). Let $\varphi$ be a state formula. The functions $t, t^{\prime}$ defined inductively below translate $\varphi$ in guarded form.

\begin{tabular}{|l||l|l|}
\hline \multicolumn{1}{|c||}{$\varphi$} & \multicolumn{1}{c|}{$t(\varphi)$} & \multicolumn{1}{c|}{$t^{\prime}(\varphi)$} \\
\hline \hline $\mathrm{F}$ & $\mathrm{F}$ & $\mathrm{F}$ \\
\hline $\mathrm{T}$ & $\mathrm{T}$ & $\mathrm{T}$ \\
\hline$\varphi_{1} \vee \varphi_{2}$ & $t\left(\varphi_{1}\right) \vee t\left(\varphi_{2}\right)$ & $t^{\prime}\left(\varphi_{1}\right) \vee t^{\prime}\left(\varphi_{2}\right)$ \\
\hline$\varphi_{1} \wedge \varphi_{2}$ & $t\left(\varphi_{1}\right) \wedge t\left(\varphi_{2}\right)$ & $t^{\prime}\left(\varphi_{1}\right) \wedge t^{\prime}\left(\varphi_{2}\right)$ \\
\hline$\langle\alpha\rangle \varphi$ & $\langle\alpha\rangle t(\varphi)$ & $\langle\alpha\rangle t(\varphi)$ \\
\hline$[\alpha] \varphi$ & {$[\alpha] t(\varphi)$} & {$[\alpha] t(\varphi)$} \\
\hline$X$ & $X$ & $X$ \\
\hline$\sigma X . \varphi$ & $\sigma X . f\left(t^{\prime}(\varphi), X, \sigma\right)$ & $f\left(t^{\prime}(\varphi), X, \sigma\right)\left[\sigma X . f\left(t^{\prime}(\varphi), X, \sigma\right) / X\right]$ \\
\hline
\end{tabular}

The interested reader can easily check that $t\left(\varphi_{1}\right)=\varphi_{3}$. The syntactic and semantic properties below state that this transformation indeed reduces formulas to guarded form while preserving their interpretation.

Proposition 2. Let $\varphi$ be a state formula. Then:

$$
g(t(\varphi), \emptyset) \wedge w g\left(t^{\prime}(\varphi), f v(\varphi)\right)
$$

where $f v(\varphi)$ is the set of free variables of $\varphi$.

Proposition 3. Let $\varphi$ be a state formula. Then:

$$
\llbracket t(\varphi) \rrbracket \rho=\llbracket t^{\prime}(\varphi) \rrbracket \rho=\llbracket \varphi \rrbracket \rho
$$

for any propositional context $\rho$.

Proof. By structural induction on $\varphi$. We show only the case $\varphi=\sigma X . \varphi_{1}$, the other cases being straightforward. By definition of $t^{\prime}$ and unfolding of fixed points, we have $\llbracket t^{\prime}\left(\sigma X . \varphi_{1}\right) \rrbracket \rho=\llbracket f\left(t^{\prime}\left(\varphi_{1}\right), X, \sigma\right)\left[\sigma X . f\left(t^{\prime}\left(\varphi_{1}\right), X, \sigma\right) / X\right] \rrbracket \rho=$ $\llbracket \sigma X . f\left(t^{\prime}\left(\varphi_{1}\right), X, \sigma\right) \rrbracket \rho=\llbracket t\left(\sigma X . \varphi_{1}\right) \rrbracket \rho$. By Proposition 1 and inductive hypothesis, it follows that $\llbracket \sigma X . f\left(t^{\prime}\left(\varphi_{1}\right), X, \sigma\right) \rrbracket \rho=\llbracket \sigma X . t^{\prime}\left(\varphi_{1}\right) \rrbracket \rho=\llbracket \sigma X . \varphi_{1} \rrbracket \rho$. 
We conclude this section by an estimation of the size $|t(\varphi)|$ (number of operators and variables in $t(\varphi)$ ). The application of $t(\varphi)$ consists of flattening and unfolding steps performed in a bottom-up manner on the fixed point subformulas of $\varphi$. Flattening does not change the size of formulas, since it simply replaces some occurrences of variables by constant boolean operators. A direct implementation of fixed point unfolding would yield, for each $\sigma X . \varphi^{\prime}$ subformula of $\varphi$, a size $\left|f\left(t^{\prime}\left(\varphi^{\prime}\right), X, \sigma\right)\left[\sigma X . f\left(t^{\prime}\left(\varphi^{\prime}\right), X, \sigma\right) / X\right]\right| \leq\left|f\left(t^{\prime}\left(\varphi^{\prime}\right), X, \sigma\right)\right|^{2}=\left|t^{\prime}\left(\varphi^{\prime}\right)\right|^{2}$, leading to an overall size $|t(\varphi)| \leq|\varphi|^{2 \cdot|\varphi|}$.

An implementation of unfolding by using factorization of common subformulas would yield, for each $\sigma X . \varphi^{\prime}$ subformula of $\varphi$, a size $\left|f\left(t^{\prime}\left(\varphi^{\prime}\right), X, \sigma\right)\left[\sigma X . f\left(t^{\prime}\left(\varphi^{\prime}\right), X, \sigma\right) / X\right]\right|=\left|f\left(t^{\prime}\left(\varphi^{\prime}\right), X, \sigma\right)\right|+\left|\sigma X . f\left(t^{\prime}\left(\varphi^{\prime}\right), X, \sigma\right)\right|$. Note that the second summand above appears only once for each subformula $\sigma X . \varphi^{\prime}$ : since $f\left(t^{\prime}\left(\varphi^{\prime}\right), X, \sigma\right)$ is guarded w.r.t. $\{X\}$, all fixed point subformulas $\sigma X . f\left(t^{\prime}\left(\varphi^{\prime}\right), X, \sigma\right)$ substituted for $X$ in $f\left(t^{\prime}\left(\varphi^{\prime}\right), X, \sigma\right)$ will occur in the scope of modalities and will remain unchanged during later flattening steps. In this way, each fixed point subformula of $\varphi$ will be duplicated only once (at the moment of its unfolding) and reused in later steps, leading to an overall size $|t(\varphi)| \leq|\varphi|^{2}$.

The translations to guarded form proposed in 15,23 . perform the flattening of a formula $\sigma X . \varphi$ by converting $\varphi$ in conjunctive normal form (considering modal and fixed point subformulas as literals) before replacing the top-level unguarded occurrences of $X$ in $\varphi$ with $\mathrm{F}$ or $\mathrm{T}$. This yields a worst-case exponential size of the final formula, even if a factorization scheme is applied.

\subsection{Simplification of Guarded Mu-Calculus on ALTSs}

We show in this section that guarded $\mu$-calculus formulas can be considerably simplified when interpreted on ALTSs $M=\left(S, A, T, s_{0}\right)$, consequently increasing the efficiency of model-checking. For technical reasons, we need to use the negation operator $(\neg)$ on state formulas. The negation of a state formula $\varphi$, noted $\neg \varphi$, is interpreted in a context $\rho$ as $\llbracket \neg \varphi \rrbracket \rho=S \backslash \llbracket \varphi \rrbracket \rho$. For the sake of simplicity, we did not use the negation operator in the definition of $\mu$-calculus given in Section 2.1. In the presence of negation, to ensure the well-definedness of the interpretation of fixed point formulas, we must impose the syntactic monotonicity condition [15]: in any fixed point formula $\mu X . \varphi$ or $\nu X . \varphi$, all free occurrences of $X$ in $\varphi$ must be in the scope of an even number of negations. Using duality (see below), any syntactically monotonic formula can be converted in positive form, i.e., an equivalent formula without negation operators.

The dual of a state formula $\varphi$ w.r.t. a set of propositional variables $\left\{X_{1}, \ldots, X_{n}\right\}$ is the negation of $\varphi$ in which all free occurrences of $X_{1}, \ldots, X_{n}$ are respectively replaced by $\neg X_{1}, \ldots, \neg X_{n}$. However, to facilitate the reasoning by structural induction, we prefer an inductive definition of dual formulas.

Definition 4 (dual formulas). Let $\varphi$ be a state formula and let $\mathcal{X}=$ $\left\{X_{1}, \ldots, X_{n}\right\}$ be a set of propositional variables. The formula $d(\varphi, \mathcal{X})$ defined inductively below is called the dual of $\varphi$ w.r.t. $\mathcal{X}$. 


\begin{tabular}{|l|l||l|l|}
\hline \multicolumn{1}{|c|}{$\varphi$} & \multicolumn{1}{c|}{$d(\varphi, \mathcal{X})$} & \multicolumn{1}{c|}{$\varphi$} & \multicolumn{1}{c|}{$d(\varphi, \mathcal{X})$} \\
\hline \hline $\mathrm{F}$ & $\mathrm{T}$ & $\mathrm{T}$ & $\mathrm{F}$ \\
\hline$\varphi_{1} \vee \varphi_{2}$ & $d\left(\varphi_{1}, \mathcal{X}\right) \wedge d\left(\varphi_{2}, \mathcal{X}\right)$ & $\varphi_{1} \wedge \varphi_{2}$ & $d\left(\varphi_{1}, \mathcal{X}\right) \vee d\left(\varphi_{2}, \mathcal{X}\right)$ \\
\hline$\langle\alpha\rangle \varphi$ & {$[\alpha] d(\varphi, \mathcal{X})$} & {$[\alpha] \varphi$} & $\langle\alpha\rangle d(\varphi, \mathcal{X})$ \\
\hline$X$ & $X$ if $X \in \mathcal{X}$ & $X$ & $\neg X$ if $X \notin \mathcal{X}$ \\
\hline$\mu X . \varphi$ & $\nu X . d(\varphi, \mathcal{X} \cup\{X\})$ & $\nu X . \varphi$ & $\mu X . d(\varphi, \mathcal{X} \cup\{X\})$ \\
\hline
\end{tabular}

Proposition 4. Let $M=\left(S, A, T, s_{0}\right)$ be an LTS, $\varphi$ be a state formula, and $X_{1}, \ldots, X_{n}$ be propositional variables. Then:

$$
S \backslash \llbracket \varphi \rrbracket \rho=\llbracket d\left(\varphi,\left\{X_{1}, \ldots, X_{n}\right\}\right) \rrbracket \rho\left[S \backslash \rho\left(X_{1}\right) / X_{1}, \ldots, S \backslash \rho\left(X_{n}\right) / X_{n}\right]
$$

for any propositional context $\rho$.

Proposition 4 allows in particular to derive the duality between minimal and maximal fixed point formulas: $S \backslash \llbracket \mu X . \varphi \rrbracket \rho=\llbracket d(\mu X . \varphi, \emptyset) \rrbracket \rho=\llbracket \nu X . d(\varphi,\{X\}) \rrbracket \rho$. Lemma 2 establishes a more involved property about dual formulas interpreted on ALtss: every state satisfying both a formula and its dual is the origin of a particular sequence of ( 0 or more) transitions in the Alts.

Lemma 2. Let $M=\left(S, A, T, s_{0}\right)$ be an Alts, $\varphi$ be a state formula, $X_{1}, \ldots, X_{n}$ be propositional variables, $A_{1}, B_{1}, \ldots, A_{n}, B_{n} \subseteq S$ be state sets, and $\mathcal{Y} \subseteq$ $\left\{X_{1}, \ldots, X_{n}\right\}$ be a set of variables such that $g(\varphi, \mathcal{Y})$. Then:

$$
\begin{aligned}
& \forall s \in \llbracket \varphi \rrbracket \rho\left[A_{1} / X_{1}, \ldots, A_{n} / X_{n}\right] \cap \llbracket d\left(\varphi,\left\{X_{1}, \ldots, X_{n}\right\}\right) \rrbracket \rho\left[B_{1} / X_{1}, \ldots, B_{n} / X_{n}\right] . \\
& \exists i \in[1, n] . \exists s^{\prime} \in A_{i} \cap B_{i} . s \stackrel{*}{\rightarrow} s^{\prime} \wedge\left(X_{i} \in \mathcal{Y} \Rightarrow s^{\prime} \neq s\right)
\end{aligned}
$$

for any propositional context $\rho$.

Theorem 1 gives a characterization of ALTSs by means of guarded $\mu$-calculus formulas: ALTSs are precisely those LTSs on which minimal and maximal guarded fixed point formulas have the same interpretation.

Theorem 1 (characterization of acyclic LTSs). Let $M=\left(S, A, T, s_{0}\right)$ be an LTS. $M$ is acyclic iff, for every state formula $\varphi$ and every propositional variable $X$ such that $g(\varphi,\{X\})$, the following equality holds:

$$
\llbracket \mu X . \varphi \rrbracket \rho=\llbracket \nu X . \varphi \rrbracket \rho
$$

for any propositional context $\rho$.

Proof. If. Let $M=\left(S, A, T, s_{0}\right)$ be an LTs, and consider the formula $\varphi=[\mathrm{T}] X$, which obviously satisfies $g(\varphi,\{X\})$. By hypothesis, we have that $\llbracket \mu X$. $[\mathrm{T}] X \rrbracket=$ $\llbracket \nu X .[\mathrm{T}] X \rrbracket=S$. This means $M \models \mu X$. [T] $X$, i.e., for every state $s \in S$, all outgoing transition sequences are finite. Hence, $M$ is acyclic.

Only if. Let $M=\left(S, A, T, s_{0}\right)$ be an Alts, $\varphi$ be a state formula, and $X$ be a propositional variable such that $g(\varphi,\{X\})$. We show that $\llbracket \nu X . \varphi \rrbracket \rho \backslash \llbracket \mu X . \varphi \rrbracket \rho=\emptyset$ for an arbitrary propositional context $\rho$. Using the duality between minimal and maximal fixed points, this equality can be rewritten as $\llbracket \nu X . \varphi \rrbracket \rho \cap$ $\llbracket \nu X . d(\varphi,\{X\}) \rrbracket \rho=\emptyset$. We note $A=\llbracket \nu X . \varphi \rrbracket \rho$ and $B=\llbracket \nu X . d(\varphi,\{X\}) \rrbracket \rho$. By 
unfolding the fixed point formulas, the above equality becomes $\llbracket \varphi \rrbracket \rho[A / X] \cap$ $\llbracket d(\varphi,\{X\}) \rrbracket \rho[B / X]=\emptyset$.

Suppose this equality does not hold, and let $s \in \llbracket \varphi \rrbracket \rho[A / X] \cap \llbracket d(\varphi,\{X\}) \rrbracket$ $\rho[B / X]$. By applying Lemma 2 , and since $g(\varphi,\{X\})$ by hypothesis, this implies $s \stackrel{*}{\rightarrow} s^{\prime}$, where $s^{\prime} \in A \cap B=\llbracket \varphi \rrbracket \rho[A / X] \cap \llbracket d(\varphi,\{X\}) \rrbracket \rho[B / X]$ and $s^{\prime} \neq s$. Thus, from every state $s \in A \cap B$ there is a non empty transition sequence to another state $s^{\prime} \in A \cap B$. Since the state set $A \cap B$ is finite, this means there is a cycle between states in $A \cap B$ (contradiction with $M$ acyclic).

The practical consequence of Theorem 11 is to allow the simplification of guarded state formulas interpreted on ALTSs by converting all occurrences of maximal fixed points into minimal fixed points (or vice-versa, which would lead to an equivalent simplification). The resulting formulas $\varphi$ are alternation-free [9] because they do not contain mutually recursive minimal and maximal fixed point operators, and consequently their model-checking on ALtss $M=\left(S, A, T, s_{0}\right)$ has a time and space complexity $O(|\varphi| \cdot(|S|+|T|))$ [5].

Together with the translation in guarded form given in Section 2.2 the reduction provided by Theorem 1 yields an overall complexity of $O\left(|\varphi|^{2} \cdot(|S|+|T|)\right)$ for the model-checking of arbitrary $\mu$-calculus formulas on ALtss. Moreover, the space complexity of model-checking can be improved in this case to $O\left(|\varphi|^{2} \cdot|S|\right)$, as shown in the next section.

\section{Local Model-Checking on Acyclic LTSs}

After applying the translation to guarded form and the simplification defined in Sections 2.2 and 2.3 , we obtain simplified guarded state formulas $\mu X . \varphi$ that contain only minimal fixed point variables. The local verification of this kind of formulas on ALTSs could be easily handled by the existing model-checking algorithms for alternation-free $\mu$-calculus [124,17/7]. However, by exploiting the absence of cycles in the AlTs and the guardedness property of the formulas, we can devise algorithms with a better space complexity.

We adopt an approach that has been used for model-checking the alternationfree $\mu$-calculus [124]. The approach consists of translating the verification problem to the local resolution of a boolean equation system, which is performed by on-the-fly exploration of the dependency graph between boolean variables.

\subsection{Translation to Boolean Equation Systems}

We encode the model-checking problem of a guarded simplified formula on an Alts by using boolean equation systems (BESs) [18. A (simple) Bes (see Table 21) is a system of minimal fixed point equations $\left\{z_{i}=o p_{i} Z_{i}\right\}_{1<i<n}$ whose left-hand sides are boolean variables $z_{i} \in \mathcal{Z}$ and whose right-hand sides are pure disjunctive or conjunctive boolean formulas. Empty disjunctions and conjunctions are equivalent to $\mathrm{F}$ and $\mathrm{T}$, respectively. The interpretation of a BES

\footnotetext{
${ }^{1}$ Any formula $\varphi$ can be converted to the form $\mu X . \varphi$, where $X$ is a "fresh" variable.
} 
w.r.t. a boolean context $\delta: \mathcal{Z} \rightarrow\{\mathrm{F}, \mathrm{T}\}$ is the minimal fixed point of a vectorial functional $\bar{\Psi}_{\delta}$ defined over $\{\mathrm{F}, \mathrm{T}\}^{n}$.

Table 2. Syntax and semantics of Bess

$$
\begin{aligned}
& \left\{z_{i}=o p_{i} Z_{i}\right\}_{1 \leq i \leq n} \\
& \text { where } o p_{i} \in\{\bigvee, \wedge\}, Z_{i} \subseteq \mathcal{Z} \text { for all } 1 \leq i \leq n \\
& \llbracket o p_{i}\left\{z_{1}, \ldots, z_{p}\right\} \rrbracket \delta=\delta\left(z_{1}\right) o p_{i} \ldots o p_{i} \delta\left(z_{p}\right) \\
& \llbracket\left\{z_{i}=o p_{i} Z_{i}\right\}_{1 \leq i \leq n} \rrbracket \delta=\mu \bar{\Psi}_{\delta} \\
& \text { where } \bar{\Psi}_{\delta}:\{\mathrm{F}, \mathrm{T}\}^{n} \rightarrow\{\mathrm{F}, \mathrm{T}\}^{n} \\
& \bar{\Psi}_{\delta}\left(b_{1}, \ldots, b_{n}\right)=\left(\llbracket o p_{i} Z_{i} \rrbracket \delta\left[b_{1} / z_{1}, \ldots, b_{n} / z_{n}\right]\right)_{1 \leq i \leq n}
\end{aligned}
$$

The function $h_{1}$ defined below constructs a BES encoding the model-checking problem of a guarded simplified formula $\mu X . \varphi$ on an ALTs. For each subformula $\varphi^{\prime}$ of $\varphi$ and each $s \in S$, the BES defines a boolean variable $Z_{\varphi^{\prime}, s}$ which is true iff $s$ satisfies $\varphi^{\prime}$. The auxiliary function $h_{2}\left(\varphi^{\prime}, s\right)$ produces the boolean formulas

\begin{tabular}{|c|c|c|}
\hline$\varphi$ & $h_{1}(\varphi, s)$ & $h_{2}(\varphi, s)$ \\
\hline $\mathrm{F}$ & \multirow{2}{*}{} & Vø \\
\hline$T$ & & $\triangle \emptyset$ \\
\hline$\varphi_{1} \vee \varphi_{2}$ & \multirow{2}{*}{$\begin{array}{l}\left\{Z_{\varphi_{1}, s}=h_{2}\left(\varphi_{1}, s\right), Z_{\varphi_{2}, s}=h_{2}\left(\varphi_{2}, s\right)\right\} \cup \\
h_{1}\left(\varphi_{1}, s\right) \cup h_{1}\left(\varphi_{2}, s\right)\end{array}$} & $Z_{\varphi_{1}, s} \vee Z_{\varphi_{2}, s}$ \\
\hline$\varphi_{1} \wedge \varphi_{2}$ & & $Z_{\varphi_{1}, s} \wedge Z_{\varphi_{2}, s}$ \\
\hline$\langle\alpha\rangle \varphi$ & \multirow{2}{*}{$\begin{array}{l}\left\{Z_{\varphi, s^{\prime}}=h_{2}\left(\varphi, s^{\prime}\right) \mid s \stackrel{a}{\rightarrow} s^{\prime} \wedge a \in \llbracket \alpha \rrbracket\right\} \cup \\
h_{1}(\varphi, s)\end{array}$} & $\bigvee\left\{Z_{\varphi, s^{\prime}} \mid s \stackrel{a}{\rightarrow} s^{\prime} \wedge a \in \llbracket \alpha \rrbracket\right\}$ \\
\hline$[\alpha] \varphi$ & & $\bigwedge\left\{Z_{\varphi, s^{\prime}} \mid s \stackrel{a}{\rightarrow} s^{\prime} \wedge a \in \llbracket \alpha \rrbracket\right\}$ \\
\hline$X$ & \{\} & \multirow[t]{2}{*}{$Z_{X, s}$} \\
\hline$\mu X . \varphi$ & $\left\{Z_{X, s}=h_{2}(\varphi, s)\right\} \cup h_{1}(\varphi, s)$ & \\
\hline
\end{tabular}
in the right-hand sides of the equations, by introducing extra variables in order to obtain only pure disjunctive or conjunctive formulas.

Definition 5 (translation to BESs). Let $\mu X . \varphi$ be a closed simplified guarded formula and $M=\left(S, A, T, s_{0}\right)$ be an Alts. The translation $h_{1}(\mu X . \varphi, s)$ defined below yields a BES encoding the satisfaction of $\mu X . \varphi$ by a state $s \in S$.

The size of the resulting BES is linear in the size of the formula $\mu X . \varphi$ and the size of the ALTS: there are at most $|\varphi| \cdot|S|$ boolean variables and $|\varphi| \cdot(|S|+|T|)$ operators in the right-hand sides. The functions $h_{1}, h_{2}$ are very similar to other translations from fixed point formulas to Bess given in [51] or [18, chap. 2]. The local model-checking problem of $\mu X . \varphi$ on the initial state of $M=\left(S, A, T, s_{0}\right)$ reduces to the resolution of variable $Z_{X, s_{0}}$ in the $\operatorname{BES} h_{1}\left(\mu X . \varphi, s_{0}\right)$.

Proposition 5. Let $M=\left(S, A, T, s_{0}\right)$ be an ALTS and $\mu X . \varphi$ be a closed, simplified guarded formula. Then:

$$
\llbracket \mu X . \varphi \rrbracket=\left\{s \in S \mid\left(\llbracket h_{1}(\mu X . \varphi, s) \rrbracket\right)_{X, s}=\mathrm{T}\right\} .
$$


Note that during the translation given in Definition 5, the transitions $s \stackrel{a}{\rightarrow} s^{\prime}$ are traversed forwards, which enables to construct the Alts simultaneously with the BES in a demand-driven way.

\subsection{Local Resolution of BESs}

For developing our resolution algorithm, we use a representation of BESs as boolean graphs [1], which provide a more intuitive way of reasoning about dependencies between boolean variables. To every $\operatorname{BES}\left\{z_{i}=o p_{i} Z_{i}\right\}_{1 \leq i \leq n}$ corresponds a boolean graph $G=(V, E, L)$, where: $V=\left\{z_{1}, \ldots, z_{n}\right\}$ is the set of vertices (boolean variables), $E=\left\{\left(z_{i}, z_{j}\right) \mid 1 \leq i, j \leq n \wedge z_{j} \in Z_{i}\right\}$ is the set of edges (dependencies between boolean variables), and $L: V \rightarrow\{\vee, \wedge\}, L\left(z_{i}\right)=o p_{i}$ is the vertex labeling (vertices are conjunctive or disjunctive according to the operator in the corresponding equation). The set of successors of a vertex $z$ is noted $E(z)$. We assume that vertices in $E(z)$ are ordered from $(E(z))_{0}$ to $(E(z))_{|E(z)|-1}$. A boolean graph is represented implicitly by the successor function, which associates to each vertex $z$ the set $E(z)$.

Note that the boolean graphs produced from BESs encoding the local modelchecking of guarded $\mu$-calculus formulas on ALTSs are acyclic (otherwise there would be either a cycle in the ALTS, or an unguarded occurrence of a propositional variable in the formula).

The DAGSolve algorithm that we propose for the local resolution of a BES with acyclic boolean graph is shown in Figure 1. It takes as input a boolean graph $G=(V, E, L)$ represented implicitly and a vertex $x \in V$ denoting a boolean variable. Starting at vertex $x$, DAGSolvE recursively performs a depthfirst search of $G$ and for each vertex $y$ encountered it computes its truth value $v(y)$. A counter $p(x)$ indicates the next successor of $x$ to be visited. The vertices already visited are stored in a global set $A \subseteq V$ (initially empty). The exploration of the successors of $x$ is stopped as soon as its truth value can be decided (e.g., if $L(x)=\vee$, then $v(x)$ becomes $\mathrm{T}$ as soon as a successor $y \in E(x)$ with $v(y)=\mathrm{T}$ has been encountered). Upon termination of the call DagSolve $(x,(V, E, L))$, vertex $x$ is contained in $A$ and its final truth value is given by $v(x)$.

DAGSOLVE is similar in spirit with other local BES resolution algorithms [1. 24,7] based upon exploration of the dependency graph between boolean variables. Like these algorithms, DAGSolve has an $O(|V|+|E|)$ time complexity, equivalent to $O(|\varphi| \cdot(|S|+|T|))$ in terms of the state formula and the Alts. However, by exploiting the absence of cycles in $G$, DAGSolve does not need to keep track of backward dependencies for updating the value of variables, and therefore it does not store the transitions of $G$ in memory. This yields for DAGSolve a space complexity $O(|V|)$, equivalent to $O(|\varphi| \cdot|S|)$, instead of $O(|V|+|E|)$ like the algorithms in [1|24/7] when they are executed on acyclic boolean graphs.

\subsection{Handling of Unguarded Alternation-Free Mu-Calculus}

To verify an alternation-free $\mu$-calculus formula $\varphi$ on an ALTs $M=\left(S, A, T, s_{0}\right)$, one could first transform $\varphi$ in simplified guarded form by using the translations 


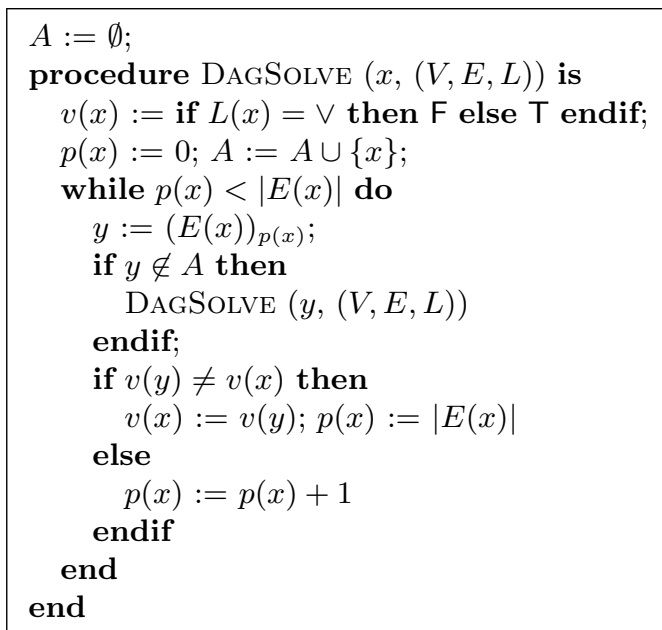

Fig. 1. Local resolution of BESs with acyclic boolean graphs

given in Sections 2.2 and 2.3. and then apply the DAgSolve model-checking algorithm given in Section [3.2. If $\varphi$ is already guarded, this procedure would yield a time complexity $O(|\varphi| \cdot(|S|+|T|))$ and a space complexity $O(|\varphi| \cdot|S|)$.

However, if $\varphi$ is unguarded, this would yield a worst-case time complexity $O\left(|\varphi|^{2} \cdot(|S|+|T|)\right)$ instead of the complexity $O(|\varphi| \cdot(|S|+|T|))$ obtained by using an existing linear-time model-checking algorithm 124/7. Although unguarded formulas are seldom in practice, and the blow-up caused by translation in guarded form is often less than quadratic, the above procedure may be unacceptable in some cases. Therefore, we search to devise a linear-time, memory efficient algorithm for checking unguarded alternation-free formulas on ALTSs.

Unguarded propositional variables occurring in a formula $\mu X . \varphi$ will induce cyclic dependencies in the corresponding BES (i.e., cycles in the boolean graph), even if the LTs $M=\left(S, A, T, s_{0}\right)$ is acyclic. However, these cycles have a rather simple structure: they contain only vertices of the form $Z_{\varphi_{1}, s}, Z_{\varphi_{2}, s}, \ldots$, where $\varphi_{1}$, $\varphi_{2}, \ldots$ are subformulas of $\varphi$ dominated by boolean or fixed point operators, and the state $s$ remains unchanged along the cycle. Vertices belonging to a strongly connected component (SCC) of the boolean graph are reachable from the vertex of interest $Z_{X, s_{0}}$ only through the root of the SCC, which is always a vertex $Z_{X^{\prime}, s}$ corresponding to a closed subformula $\mu X^{\prime} . \varphi^{\prime}$. Moreover, assuming there is no factorization of common subformulas in the initial formula $\mu X . \varphi$, a vertex of a SCC is reachable from the root of the SCC via a single path.

The AfmcSolve algorithm that we propose for solving these BESs is shown in Figure 2, We consider here only the case of minimal fixed point BESs, the other case being dual (every alternation-free formula can be checked by combining these two algorithms [120]). Besides an implicit boolean graph $G=(V, E, L)$ 
and a vertex $x \in V$, AfmcSolve takes as input a predicate root $: V \rightarrow\{\mathrm{F}, \mathrm{T}\}$ indicating the roots of the ScCs of $G$.

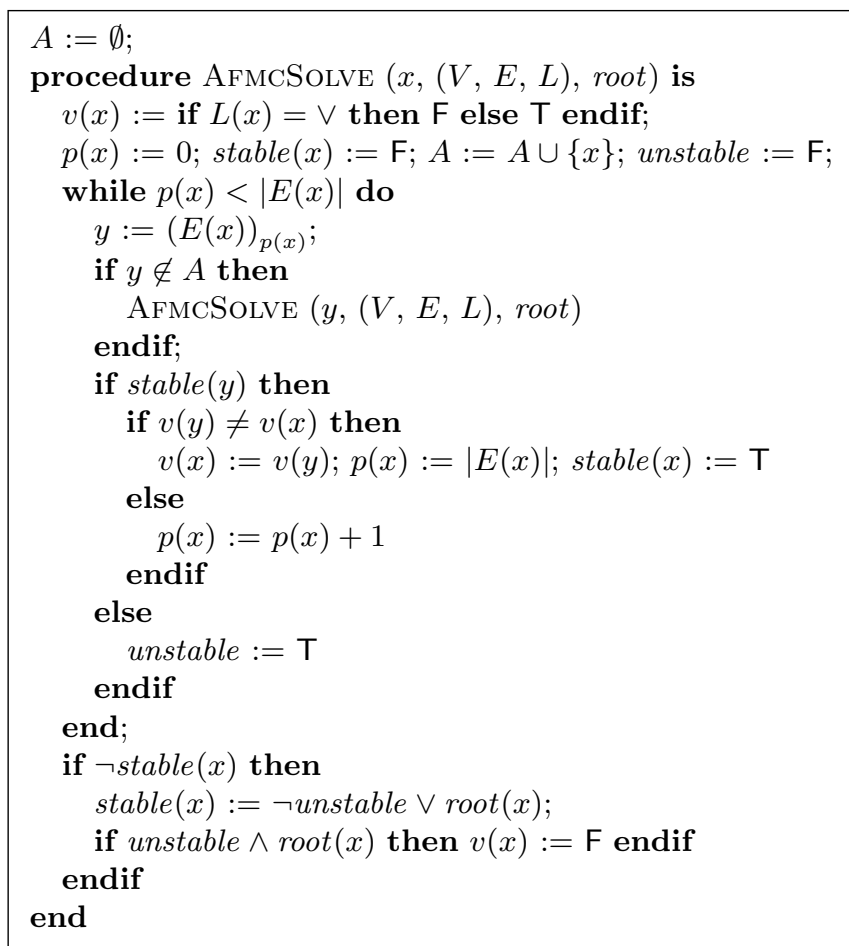

Fig. 2. Local resolution of BESs produced from unguarded alternation-free formulas

AfmcSolve is similar to DAgSolve, except that the value $v(x)$ of some vertices $x$ (which have an outgoing path leading to an "ancestor" vertex currently on the call stack of AFMCSOLVE) cannot be decided upon termination of the call $\operatorname{AfmCSOLVE}(x,(V, E, L)$, root). This information is recorded using an additional boolean stable $(x)$, which is set to $\mathrm{T}$ in the following situations: (a) $v(x)$ has been decided after exploring a stable successor $y \in E(x)$; (b) $v(x)$ has not been decided after exploring all successors in $E(x)$, but either no unstable successor of $x$ has been encountered, or $x$ is the root of a SCC of $G$ (in this last case, $v(x)$ is set to $\mathrm{F}$, since $G$ denotes a minimal fixed point BES).

After deciding $v(x)$, there is no need to propagate this value to the "descendants" of $x$ already explored, because these vertices will not be reached anymore in the ScC of $x$. Therefore, AfmcSolve does not need to store the transitions of $G$ in memory, achieving a space complexity $O(|V|)$, equivalent to $O(|\varphi| \cdot|S|)$. 


\section{Conclusion and Future Work}

We showed that full modal $\mu$-calculus interpreted on acyclic LTSs has the same expressive power as its alternation-free fragment. We also proposed two local model-checking algorithms: DAGSOLVE, which handles formulas $\varphi$ with alternation depth $\operatorname{ad}(\varphi) \geq 2$ and has time complexity $O\left(|\varphi|^{2} \cdot(|S|+|T|)\right)$ and space complexity $O\left(|\varphi|^{2} \cdot|S|\right)$; and AFmCSolve, which handles alternation-free formulas and has time complexity $O(|\varphi| \cdot(|S|+|T|))$ and space complexity $O(|\varphi| \cdot|S|)$. Both algorithms have been implemented using the generic OPEN/CÆSAR environment 12 for on-the-fly exploration of LTSs, and are integrated within the Evaluator 3.5 model-checker [20] of the CADP verification toolbox [10].

These results are currently applied in an industrial project involving the verification and testing of multiprocessor architectures designed by BuLL. One of the verification tasks concerns the off-line analysis of large execution traces (about 100,000 events) produced by intensive testing of a hardware cache coherency protocol. Several hundreds correctness properties, derived from the formal specification of the protocol, are checked on the set of traces (grouped in one ALTS). Most properties are expressed as PDL 11 formulas $\left[R_{1}\right]\left\langle R_{2}\right\rangle \mathrm{T}$, where $R_{1}$ and $R_{2}$ are complex regular expressions encoding sequences of request and response actions. These formulas are translated in guarded alternation-free $\mu$-calculus, simplified by replacing maximal fixed points with minimal ones, and checked on the Alts using DAGSolve. Compared to standard algorithms for alternation-free $\mu$-calculus, this procedure improves execution time (the number of ALTs traversals is reduced) and memory consumption (ALTs transitions are not stored).

The model-checking approach we proposed can be directly applied to other forms of trace analysis (e.g., run-time monitoring, security log file auditing, etc.) by encoding these problems as model-checking of temporal formulas on single trace ALTSs. Moreover, our simplification of guarded $\mu$-calculus interpreted on Altss (equivalence between minimal and maximal fixed points) can be useful as an intermediate optimization step in other model-checkers, by allowing to simplify temporal formulas in a similar manner when verifying ALTss. For instance, when checking CTL 3 formulas on Altss, our simplification makes valid the equality $\mathrm{A}\left[\varphi_{1} \mathrm{U} \varphi_{2}\right]=\neg \mathrm{E}\left[\neg \varphi_{2} \mathrm{U} \neg\left(\varphi_{2} \vee\left(\varphi_{1} \wedge \mathrm{EXT}\right)\right)\right]$, which allows to derive all CTL operators from E[.U.] and to reduce the model-checking of CTL to the search of finite transition sequences satisfying $\mathrm{E}\left[\varphi_{1} \cup \varphi_{2}\right]$.

Two directions for future work seem promising. Firstly, one could devise a local model-checking algorithm for guarded $\mu$-calculus that works without backtracking in the AlTs (this is not guaranteed by DAGSOLVE, which uses a depthfirst traversal of the boolean graph). When analyzing a trace in real-time during its generation, this algorithm would scan it only once, potentially leading to performance improvements. Secondly, our model-checking procedure could be used for comparing on-the-fly an LTS $M_{1}$ with an ALTS $M_{2}$ modulo a bisimulation or preorder relation. This problem has various practical applications (search for execution sequences, interactive replay of simulation trees and model-checking diagnostics, etc.) and could be solved by building a guarded characteristic formula [14] of $M_{1}$ and verifying it on $M_{2}$ using the DAGSolve algorithm. 


\section{References}

1. H. R. Andersen. Model checking and boolean graphs. Th. Co. Sci., 126:3-30, 1994.

2. J. C. Bradfield and C. Stirling. Modal Logics and Mu-Calculi: an Introduction. In Handbook of Process Algebra, pp. 293-330, Elsevier, 2001.

3. E. Clarke, O. Grumberg, and D. Peled. Model Checking. MIT Press, 2000.

4. R. Cleaveland, M. Klein, and B. Steffen. Faster Model Checking for the Modal Mu-Calculus. In $C A V^{\prime} 92$, LNCS vol. 663, pp. 410-422.

5. R. Cleaveland and B. Steffen. A Linear-Time Model-Checking Algorithm for the Alternation-Free Modal Mu-Calculus. FM in Syst. Design, 2:121-147, 1993.

6. F. Dietrich, X. Logean, S. Koppenhoefer, and J-P. Hubaux. Testing Temporal Logic Properties in Distributed Systems. In IWTCS'98, pp. 247-262, Kluwer, 1998.

7. X. Du, S. A. Smolka, and R. Cleaveland. Local Model Checking and Protocol Analysis. Springer STTT Journal, 2(3):219-241, 1999.

8. M. Ducassé. OPIUM: An Extendable Trace Analyzer for Prolog. Journal of Logic Programming, 39(1-3):177-224, 1999.

9. E. A. Emerson and C-L. Lei. Efficient Model Checking in Fragments of the Propositional Mu-Calculus. In $L I C S^{\prime} 86$, pp. 267-278, IEEE, 1986.

10. J-C. Fernandez, H. Garavel, A. Kerbrat, R. Mateescu, L. Mounier, and M. Sighireanu. CADP (CÆSAR/ALDEBARAN Development Package): A Protocol Validation and Verification Toolbox. In $C A V^{\prime} 96$, LNCS vol. 1102, pp. 437-440.

11. M. J. Fischer and R. E. Ladner. Propositional Dynamic Logic of Regular Programs. Journal of Computer and System Sciences, (18):194-211, 1979.

12. H. Garavel. OPEN/CÆSAR: An Open Software Architecture for Verification, Simulation, and Testing. In TACAS'98, LNCS vol. 1384, pp. 68-84. Full version available as INRIA Research Report RR-3352.

13. K. Ilgun, R. A. Kemmerer, and P. A. Porras. State Transition Analysis: A RuleBased Intrusion Detection Approach. IEEE Tr. on Soft. Eng., 21(3):181-199, 1995.

14. A. Ingolfsdottir and B. Steffen. Characteristic Formulae for Processes with Divergence. Information and Computation, 110(1):149-163, June 1994.

15. D. Kozen. Results on the Propositional $\mu$-calculus. Th. Co. Sci., 27:333-354, 1983.

16. K. G. Larsen. Efficient Local Correctness Checking. In $C A V^{\prime} 92$, LNCS vol. 663, pp. $30-43$.

17. X. Liu, C. R. Ramakrishnan, and S. A. Smolka. Fully Local and Efficient Evaluation of Alternating Fixed Points. In TACAS'98, LNCS vol. 1384, pp. 5-19.

18. A. Mader. Verification of Modal Properties Using Boolean Equation Systems. VERSAL 8, Bertz Verlag, Berlin, 1997.

19. Z. Manna and A. Pnueli. The Temporal Logic of Reactive and Concurrent Systems, volume I (Specification). Springer Verlag, 1992.

20. R. Mateescu and M. Sighireanu. Efficient On-the-Fly Model-Checking for Regular Alternation-Free Mu-Calculus. To appear in Science of Comp. Programming, 2002.

21. P. Stevens and C. Stirling. Practical Model-Checking Using Games. In TACAS'98, LNCS vol. 1384, pp. 85-101.

22. C. Stirling. Modal and Temporal Properties of Processes. Springer Verlag, 2001.

23. I. Walukiewicz. A Complete Deductive System for the $\mu$-calculus. In LICS'93, pp. 136-147. Full version available as BRICS Research Report RS-95-6, 1995.

24. B. Vergauwen and J. Lewi. Efficient Local Correctness Checking for Single and Alternating Boolean Equation Systems. In ICALP'94, LNCS vol. 820, pp. 304-315. 